\title{
Pd(II)-mediated oxidative amination for access to a 9-azabicyclo[4.2.1]nonane compound library and anatoxin-a
}

Rafid S. Dawood, a Suresh R. Chidipudi, a Daniel C. O'Connor, a William Lewis, a Daniel Hamza, ${ }^{b}$ Christopher A. Pearce, ${ }^{b}$ Gerrant

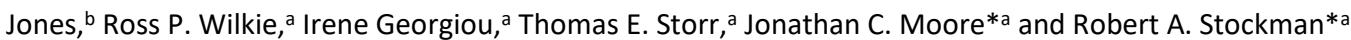

\author{
a) School of Chemistry, University Park, University of Nottingham, Nottingham, NG7 2RD, UK. E-mail: \\ robert.stockman@nottingham.ac.uk; Fax: +44 (0)115951356; \\ b) Sygnature Discovery Limited, BioCity, Pennyfoot Street, Nottingham, NG7 1GF, UK.
}

Intramolecular oxidative amination of readily accessible aminocyclooct-4-enes provides rapid and regioselective access to the 9-azabicyclo[4.2.1]nonane framework characteristic of the natural product anatoxin-a (1). This has enabled the synthesis of $\mathbf{s p}^{3}$-rich chemical scaffolds suitable for diversification. A library of 881 lead-like compounds is reported alongside a formal synthesis of anatoxin-a (1).

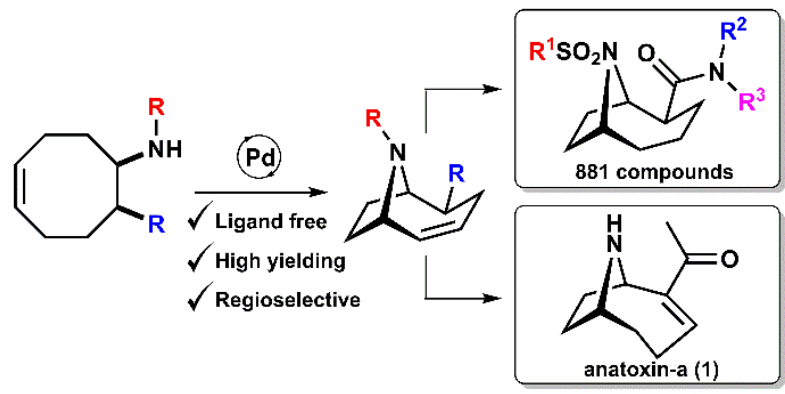

The structure of anatoxin-a (1) was unambiguously determined in 1977 through the combination of X-ray crystallographic ${ }^{1}$ and spectroscopic ${ }^{2}$ analysis. This potent neurotoxin is produced by certain strains of the freshwater blue-green algae Anabena flos-aquae and has been responsible for fatal poisoning of animal life. ${ }^{3}$ Also known as "Very Fast Death Factor", anatoxin-a (1) causes death by respiratory paralysis with an $\mathrm{LD}_{50}$ (intraperitoneal, mouse) of $0.2 \mathrm{mg} / \mathrm{kg}$, with a survival time of 4-7 minutes. ${ }^{4}$ The natural compound acts as a mimic for acetylcholine and is a potent and irreversible agonist for the nicotinic acetylcholine receptor (nAChR). ${ }^{5}$ As acetylcholine deficiency is implicated in diseases such as Alzheimer's, analogues of anatoxin-a (1) possessing lower levels of toxicity may have potential in the treatment of neurological disorders. ${ }^{6}$ Paired with its significant biological properties, the compound's unusual structure has prompted much synthetic interest; a number of distinct approaches to anatoxin-a (1) and its analogues have been devised. ${ }^{7}$ Since the topic was reviewed in $2006,{ }^{8}$ there have been a considerable number of total/formal syntheses reported including our own two-directional approach ${ }^{9}$ and a contemporary example (2016) that utilises a novel formal amide insertion strategy. ${ }^{10}$ One commonly employed approach is to construct the 9-azabicyclo[4.2.1]nonane framework through the ring closure of an aminocyclooct-4-ene (Fig. 1), however there are drawbacks to each of the methods reported to date.

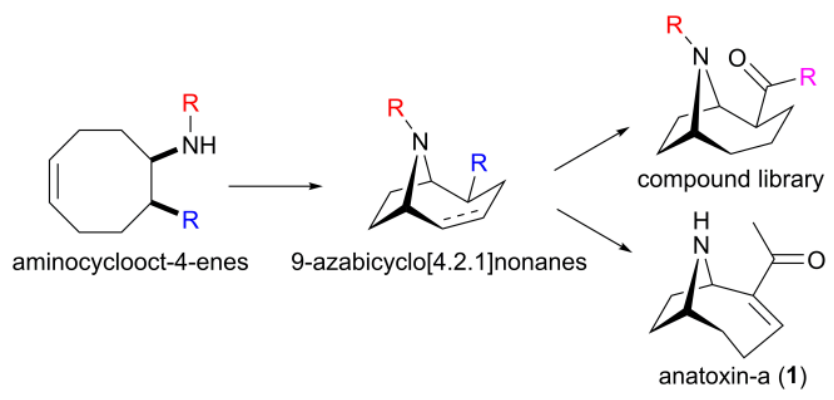

Fig. 1 - Ring closure of aminocyclooct-4-enes for the synthesis of anatoxin-a (1) and chemical scaffolds for library generation.

An aminocarbonylation strategy was reported by Ham in 1998, ${ }^{11}$ however this heterocyclisation was observed not to be regioselective. A series of reports by Parsons ${ }^{12}$ saw the development of a selenium-mediated ring closure, however this approach requires stoichiometric quantities of toxic phenylselenyl chloride. Ho ${ }^{13}$ reported a catalytic palladium mediated cyclisation of a corresponding trans-derivative, although yields were hampered by the formation of an undesired tricyclic side-product.

As part of our studies into the synthesis of chemical scaffolds for drug discovery, ${ }^{14}$ we set out to develop a catalytic and selective protocol for the heterocyclisation of aminocyclooct-4-enes. It was envisaged that a regioselective and high-yielding protocol would expediate entry to series of 9-azabicyclo[4.2.1]nonane derived chemical scaffolds that could be further derivatised to generate a lead-like compound library. Small molecule libraries are highly desired in early-stage probe- and drug-discovery screening programmes ${ }^{15}$ and as part of our work under the European Lead Factory ${ }^{16}$ we have been engaged in the development of facile synthetic routes to chemical scaffolds that are suitable 
for diversification into high $\mathrm{Fsp}^{3}$ (fraction of $\mathrm{sp}^{3-}$ hybridised carbon atoms) ${ }^{17}$ compound libraries. For these purposes we were drawn to the simplicity and concise nature of the heterocyclisation approach to the 9azabicyclo[4.2.1]nonane framework. We set out to investigate ring closure of the key precursor 2 under classical oxidative amination conditions with $\mathrm{Pd}(\mathrm{OAc})_{2}$, using $\mathrm{Cu}(\mathrm{OAc})_{2}$ as the terminal oxidant (Table 1).

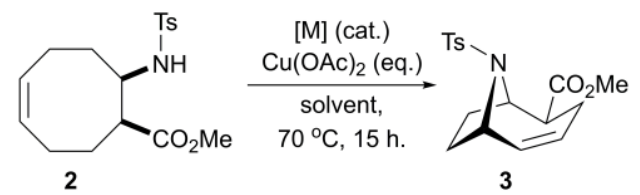

\begin{tabular}{ccccc}
\hline Entry & {$[\mathrm{M}](\mathrm{mol} \%)$} & $\begin{array}{c}\mathrm{Cu}(\mathrm{OAc})_{2} \\
\text { (eq.) }\end{array}$ & Solvent & Yield \\
\hline 1 & $\mathrm{Pd}(\mathrm{OAc})_{2}(5)$ & 2.1 & $\mathrm{THF}$ & $30 \%$ \\
2 & $\mathrm{Pd}(\mathrm{OAc})_{2}(5)$ & 2.1 & $\mathrm{PhMe}$ & $37 \%$ \\
3 & $\mathrm{Pd}(\mathrm{OAc})_{2}(5)$ & 2.1 & $\mathrm{MeCN}$ & $95 \%$ \\
4 & $\mathrm{Pd}(\mathrm{OAc})_{2}(5)$ & 2.1 & $\mathrm{DMF}$ & $99 \%$ \\
5 & $\mathrm{Pd}(\mathrm{OAc})_{2}(2.5)$ & 2.1 & $\mathrm{DMF}$ & $43 \%$ \\
6 & - & 2.1 & $\mathrm{DMF}$ & - \\
7 & {$\left[\mathrm{RuCl}\left(p-\mathrm{Cymene}_{2}\right)\right]_{2}(2.5)$} & 2.5 & $\mathrm{DMF}$ & - \\
8 & {$\left[\mathrm{RhCp} * \mathrm{Cl}_{2}\right]_{2}(2.5)$} & 2.5 & $\mathrm{DMF}$ & -
\end{tabular}

Table 1 - Catalyst/solvent optimisation. Reactions were performed on a $0.1 \mathrm{mmol}$ scale. Yields were determined by quantitative ${ }^{1} \mathrm{H}$ NMR spectroscopy with 1,3,5-trimethoxybenzene as an internal standard.

Moderate yields of the desired product 3 were obtained using THF and PhMe as solvents $(30 \%$ and $37 \%$ respectively). Changing to MeCN (Entry 3) gave an excellent conversion but DMF was found to be the superior solvent for this transformation, providing access to the 9-azabicyclo[4.2.1]nonane ring system with complete selectivity and quantitative conversion (Entry 4). Reducing the palladium loading to $2.5 \mathrm{~mol} \%$ had a significantly detrimental effect on the yield (43\%) and when the reaction was performed in the absence of palladium (Entry 6 ) or in the presence of Ru(II) and Rh(III) complexes (Entries 7-8), no cyclisation products could be detected. With the optimised conditions in hand, the substrate scope of the transformation was investigated (Fig. 2).

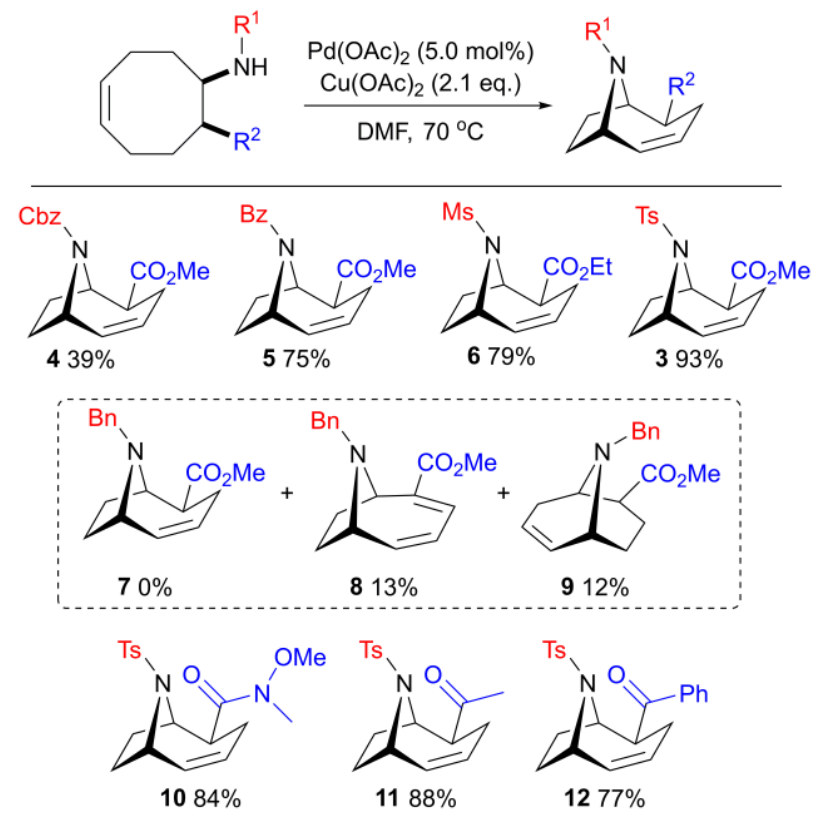


Fig. 2 - Investigation into the substrate scope of the transformation. Yields are for isolated material obtained following column chromatography.

Initially we attempted exchanging the $\mathrm{N}$-tosyl functionality for the more readily de-protected carboxybenzyl (Cbz) group. In this case, the desired product 4 was obtained in a moderate yield of $39 \%$. Benzoyl protection was considerably better tolerated, yielding the desired product $\mathbf{5}$ in a much improved $75 \%$. The electron rich $\mathrm{N}$-benzyl substrate was observed to react with poor selectivity and the targeted 9-azabicyclo[4.2.1]nonane 7 could not be isolated from the complicated mixture of products. We were however able to obtain the over-oxidised dienyl derivative 8 and the bicyclo[3.3.1]nonane derivative 9, albeit in modest yields in each case. Sulfonamides were found to be the highest yielding and most selective substrates, which is consistent with reports from the literature on many other palladium-mediated alkene aminations. ${ }^{18}$ The $N$-mesyl and -tosyl derivatives $\mathbf{6}$ and $\mathbf{3}$ were obtained in $79 \%$ and $93 \%$ respectively. In light of these results, $N$-tosylated precursors were selected to further investigate the substrate scope of the transformation with regard to the $R^{2}$ substituent (Fig. 2). We were delighted to obtain a good yield of $84 \%$ for the Weinreb amide functionalised derivative 10, which could likely serve as a useful intermediate for the synthesis of side chain-modified anatoxin-a (1) derivatives. Performing alkylation prior to ring closing was also demonstrated to be a viable strategy, with the methyl and phenyl ketones 11 and 12 being isolated in $88 \%$ and $77 \%$ respectively.

It was deemed prudent to focus the efforts of library synthesis on the high yielding and selective $N$-sulfonyl substrates. Furthermore, it was anticipated that the inherent stability of the sulfonamide functionality would help safeguard against the expected toxicity of the $\mathrm{N}$-H derivatives. It was decided that the strategy for library synthesis would involve a series of sulfonylation reactions for decoration of the first point of diversity, with $\mathrm{R}^{2}$ becoming the main diversity generating vector to be explored. As such, the $\beta$-amino esters $13 / 14$ were prepared according to a modified procedure of $\mathrm{Choi}^{19}$ and then treated with a range of sulfonyl chlorides. The selected examples represent alkyl, aryl (electron poor and rich) and hetro-aryl sulfonamide substitutions (Scheme 1, A-E). In each case sulfonylation proceeded smoothly and the products $\mathbf{2} / \mathbf{1 5 b}$-e were isolated in $\mathbf{7 6 - 9 9 \% ~ y i e l d . ~ U n d e r ~ o u r ~ o p t i m i s e d ~ c o n d i t i o n s , ~ t h e ~ o x i d a t i v e ~ a m i n a t i o n ~ o f ~} \mathbf{2} / \mathbf{1 5} \mathbf{b}-\mathbf{b}$ gave excellent yields (79-93\%) and regioselectivities (>20:1).

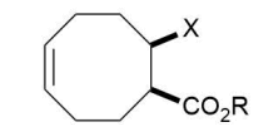

$13 \mathrm{X}=\mathrm{NH}_{3} \mathrm{Cl} ; \mathrm{R}=\mathrm{Me}$

$14 X=\mathrm{NH}_{2} ; \mathrm{R}=\mathrm{Et}$

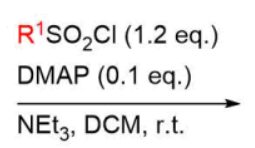

$\begin{aligned} & 2 R=M e ; R^{1}=A ; 94 \% \\ & 15 b R=E t ; R^{1}=B ; 76 \% \\ & 15 c R=E t ; R^{1}=C ; 83 \% \\ & 15 d R=E t ; R^{1}=D ; 99 \% \\ & 15 e R=E t ; R^{1}=E ; 83 \%\end{aligned}$

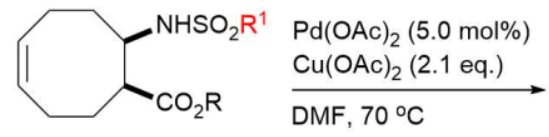

DMF, $70^{\circ} \mathrm{C}$
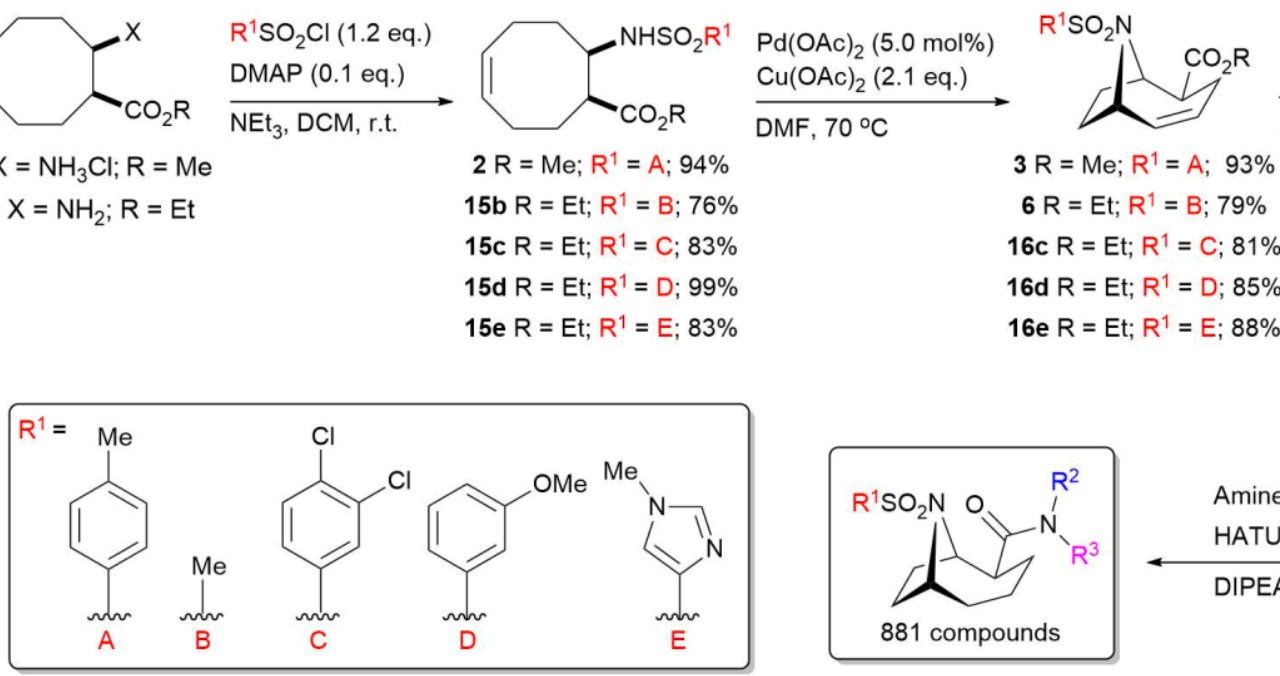

$3 \mathrm{R}=\mathrm{Me} ; \mathrm{R}^{1}=\mathrm{A} ; 93 \%$

$6 \mathrm{R}=\mathrm{Et} ; \mathrm{R}^{1}=\mathrm{B} ; 79 \%$

$16 c \mathrm{R}=\mathrm{Et} ; \mathrm{R}^{1}=\mathrm{C} ; 81 \%$

16d R = Et; $R^{1}=\mathrm{D} ; 85 \%$

16e $R=E t ; R^{1}=E ; 88 \%$

1) $\mathrm{Pd} / \mathrm{C}(10 \%$ w/w $)$
$\mathrm{H}_{2}(1 \mathrm{~atm}$.), EtOAc, r.t.
2) $\mathrm{NaOH}, \mathrm{H}_{2} \mathrm{O} / \mathrm{THF}$, r.t.

(1)

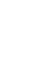

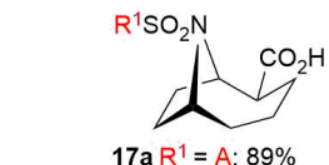

$17 b R^{1}=B ; 85 \%$

$17 \mathrm{c} \mathrm{R}^{1}=\mathrm{C} ; 95 \%$

17d R ${ }^{1}=\mathrm{D} ; 75 \%$

17 e $R^{1}=E ; 72 \%$

HATU (1.1 eq.)

DIPEA, DMF, r.t.

Scheme 1 - The synthesis and derivatisation of chemical scaffolds for the generation of a 9-azabicyclo[4.2.1]nonane compound library.

Next, to increase the "lead-likeness" of the final library the metabolically labile alkene functionality was removed via hydrogenation over palladium on carbon. In the penultimate step of library synthesis, base-mediated ester hydrolysis was employed to yield the carboxylic acid functionalised scaffolds 17 a-e in good to excellent yields (72-95\% over two steps).

With the acids 17a-e in hand, the feasibility of a library synthesis via HATU-mediated amidation in plate-format was investigated. From the 5 scaffolds 17a-e, 15 test reactions were performed using a selection of cyclic, acyclic and aromatic amines (See Supporting Information). The reaction mixtures were analysed by UPLC, filtered and purified by preparative HPLC, with purities of $85-100 \%$ as determined by LCMS. The stability of the amidation products was assessed by comparison of the ${ }^{1} \mathrm{H}$ NMR spectra obtained before and after storage in DMSO- $d_{6}$ at r.t. for 7 days. In 
each case no significant change could be observed. By utilising the general strategy for library synthesis described herein, a library of 881 9-azabicyclo[4.2.1]nonane derivatives were produced (ca.0.1 mmol each) to be incorporated into the European Lead Factory's screening programme. Computational assessment of the library indicates that it is largely Lipinski's rule of five compliant, with an average molecular weight of 445.6 Da and cLogP of 2.48. The library also has a high degree of three-dimensionality and bond-saturation, with an average Fsp ${ }^{3}$ of 0.56 (Fig. 3). In recent years there has been increased recognition of the importance of Fsp ${ }^{3}$ when designing compound libraries for lead identification. Increased bond saturation in general, as a measure of complexity, has been shown to correlate well with clinical success as compounds transition through the drug-discovery process. ${ }^{17}$ With an average $\mathrm{Fsp}^{3}$ of 0.56 , this library compares favourably to compounds in the discovery phase (average $\mathrm{Fsp}^{3}$ $=0.36$ ) and to marketed drugs (average $\left.\mathrm{Fsp}^{3}=0.47\right) \cdot{ }^{17}$
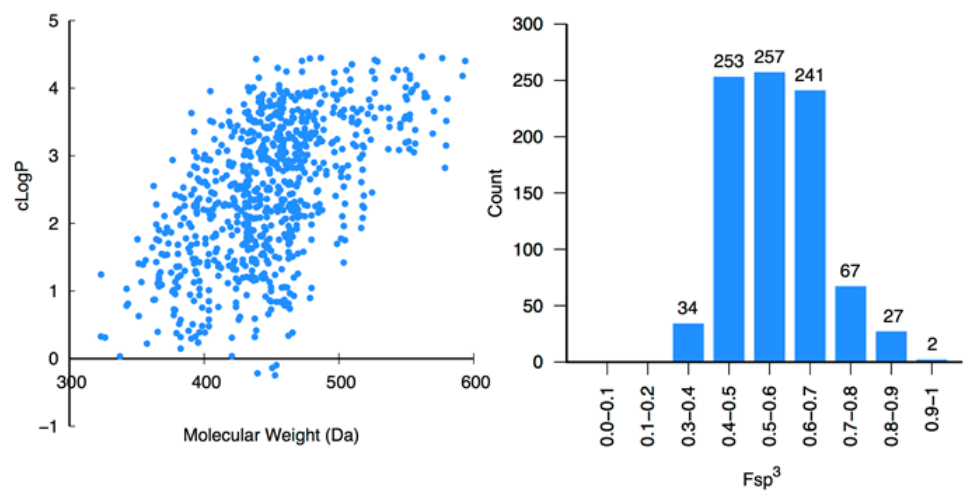

Fig. 3 - cLogP vs MW (left) and Fsp ${ }^{3}$ (right) plots of the produced 881 membered compound library.

We were also compelled to apply the oxidative amination conditions to the synthesis of anatoxin-a (1). Parsons' 2000 synthesis $^{12 a}$ utilised a selenium-mediated ring closure of the $\mathrm{N}$-Boc methyl ketone 18 to construct the 9-azabicyclo[4.2.1]nonane framework (Scheme 2). The intermediate selenide was not isolated but instead treated with $\mathrm{H}_{2} \mathrm{O}_{2}$, promoting oxidation and elimination to yield the key aza-bridged bicycle $\mathbf{1 9}$ in $55 \%$ over two steps. Interestingly, despite the report that $\mathrm{PdCl}_{2}, \mathrm{Pd}(\mathrm{OAc})_{2}$ and $\mathrm{RhCl}_{3}$ were unsuccessful at bringing about the heterocyclisation of $\mathbf{1 8}$, we were delighted to observe that utilisation of our optimised oxidative amination conditions allowed the isolation of 19 in a much improved $86 \%$ yield. This was achieved in a single synthetic operation and constitutes a useful refinement to the seminal work of Parsons ${ }^{12 a}$ and Rapoport. ${ }^{7 b}$

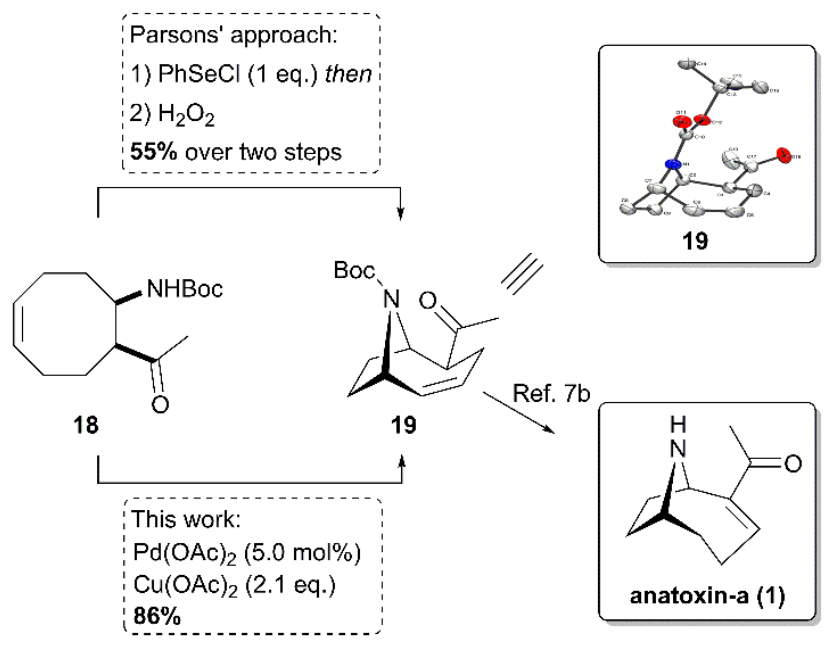

Scheme 2 - Application of the optimised oxidative amination conditions to the formal synthesis of anatoxin-a (1).

In conclusion, an improved method for the intramolecular oxidative amination of aminocyclooct-4-enes is reported for formation of the biologically relevant 9-azabicyclo[4.2.1]nonane framework. The reaction is generally high yielding, has good substrate scope and proceeds under a cheap and "ligand-free" catalytic manifold. The protocol was applied to the synthesis of a series of chemical scaffolds, which were further derivatised to form a lead-like 881 
membered compound library. The oxidative amination methodology was then applied to a formal synthesis of anatoxin-a (1), facilitating an improved yield and increased operational simplicity.

\section{Acknowledgements}

This research was done within the European Lead Factory and has received support from the Innovative Medicines Initiative Joint Undertaking (grant no. 115489), with financial contribution from the European Union's Seventh Framework Programme (FP7/2007-2013) and EFPIA companies' in-kind contribution. The authors would like to thank the University of Nottingham and the Ministry of Higher Education and Scientific Research-Iraq (MOHESR) for funding support, Professor Christopher Moody and Tom Mclnally for useful insight and discussion, Megan Thomsett and Pritesh Tailor for assistance with the library data and Thomas Ward for his synthetic contributions.

\section{Notes and references}

1 C. S. Huber, Acta Crystallogr., Sect. B 1972, 78, 2577-2582.

J. P. Devlin, O. E. Edwards, P. R. Gorham, N. R. Hunter, R. K. Pike and B. Stavric, Can. J. Chem. 1977, 55, 1367-1371. a) W. W. Carmichael, D. F. Biggs and P. R. Gorham, Science 1975, 187, 542-544; b) L. Krienitz, A. Ballot, K. Kotut, C. Wiegand, S. Pütz, J. S. Metcalf, G. A. Codd and S. Pflugmacher, FEMS Microbiol. Ecol. 2003, 43, 141-148; c) B. Puschner, B. Hoff and E. R. Tor, J. Vet. Diagnostic Investig. 2008, 20, 89-92.

4 K. L. Swanson, H. Rapoport, R. S. Aronstam and E. X. Albuquerque, Acs Symp. Ser. 1990, 418, 107-118.

5 C. E. Spivak, B. Witkop and E. X. Albuquerque, Mol. Pharmacol. 1980, 18, 384-394.

6 S. Wonnacott and T. Gallagher, Mar. Drugs 2006, 4, 228-254.

7 For total/formal syntheses of anatoxin-a, see references 8-10 and further references therein. For approaches to anatoxin derivatives please see: a) N. A. Magnus, L. Ducry, V. Rolland, S. Wonnacott and T. Gallagher, J. Chem. Soc. Trans. 1 1997, 2313-2318; b) F. J. Sardina, M. H. Howard, A. M. P. Koskinen and H. Rapoport, J. Org. Chem. 1989, 54, 4654-4660; c) P. Thomas, P. A. Brough, T. Gallagher and S. Wonnacott, Drug Dev. Res. 1994, 31, 147-156; d) D. Simoni, R. Rondanin, P. Marchetti, C. Rullo, R. Baruchello, G. Grisolia, G. Barbato, R. Giovannini, C. Marchioro, A. M. Capelli, C. Virginio, A. Bozzoli, P. A. Borea, S. Merighi and D. Donati, Bioorg. Med. Chem. Lett. 2011, 21, 54235427; e) D. B. Kanne and L. G. Abood, J. Med. Chem. 1988, 31, 506-509; f) F. I. Carroll, X. Hu, H. A. Navarro, J. Deschamps, G. R. Abdrakhmanova, M. I. Damaj and B. R. Martin, J. Med. Chem. 2006, 49, 3244-3250; g) E. Wright, T. Gallagher, C. G. V. Sharples and S. Wonnacott, Bioorg. Med. Chem. Lett. 1997, 7, 2867-2870; h) H. Gohlke, S. Schwarz, D. Gündisch, M. C. Tilotta, A. Weber, T. Wegge and G. Seitz, J. Med. Chem. 2003, 46, 2031-2048; i) M. H. Howard, F. J. Sardina and H. Rapoport, J. Org. Chem. 1990, 55, 2829-2838. For reviews on synthetic approaches to anatoxin-a and its derivatives see: a) H. L. Mansell, Tetrahedron 1996, 52, 6025-6061; b) V. Rodríguez, S. Moura, E. Pinto, C. M. P. Pereira and R. C. Braga, Quim. Nova 2006, 29, 1365-1371. a) S. J. Roe and R. A. Stockman, Chem. Commun. 2008, 3432-3434; b) S. J. Roe, D. L. Hughes, P. Aggarwal and R. A. Stockman, Synthesis 2009, 3775-3784.

10 M. Kono, S. Harada, Y. Hamada and T. Nemoto, Tetrahedron 2016, 72, 1395-1399.

11 C. -Y. Oh, K. -S. Kim and W. -H. Ham, Tetrahedron Lett. 1998, 39, 2133-2136.

12 a) P. J. Parsons, N. P. Camp, N. Edwards and L. R. Sumoreeah, Tetrahedron 2000, 56, 309-315; b) P. J. Parsons, N. P. Camp, J. M. Underwood and D. M. Harvey, Tetrahedron 1996, 52, 11637-11642; C) P. J. Parsons, N. P. Camp, J. M. Underwood and D. M. Harvey, J. Chem. Soc. Commun. 1995, 1461-1462. T. -L. Ho and E. Zinurova, Helv. Chim. Acta 2006, 89, 134-137.

a) M. J. Rawling, T. E. Storr, W. A. Bawazir, S. J. Cully, W. Lewis, M. S. I. T. Makki, I. R. Strutt, G. Jones, D. Hamza and R. A. Stockman, Chem. Commun. 2015, 51, 12867-12870; b) T. E. Storr, S. J. Cully, M. J. Rawling, W. Lewis, D. Hamza, G. Jones and R. A. Stockman, Bioorg. Med. Chem. 2015, 23, 2621-2628; c) R. S. Dawood, I. Georgiou, R. P. Wilkie, W. Lewis and R. A. Stockman, Chem. Eur. J. 2017, 23, 11153-11158; d) S. J. Cully, T. E. Storr, M. J. Rawling, I. R. Abeysena, D. Hamza, G. Jones, C. A. Pearce, A. Quddus, W. Lewis and R. A. Stockman, Bioorg. Med. Chem. 2016, 24, 5249-5257.

15 a) J.-L. Reymond and M. Awale, ACS Chem. Neurosci. 2012, 3, 649-657; b) A. W. Hung, A. Ramek, Y. Wang, T. Kaya, J. A. Wilson, P. A. Clemons and D. W. Young, Proc. Natl. Acad. Sci. U. S. A. 2011, 108, 6799-6804; c) J.-L. Reymond, R. van Deursen, L. C. Blum and L. Ruddigkeit, Med. Chem. Commun. 2010, 1, 30-38. European Lead Factory, https://www.europeanleadfactory.eu, (accessed 12 February 2018). F. Lovering, J. Bikker and C. Humblet, J. Med. Chem. 2009, 52, 6752-6756. E. M. Beccalli, G. Broggini, M. Martinelli and S. Sottocornola, Chem. Rev. 2007, 107, 5318-5365. W. Lee, S. Kwon, P. Kang, I. A. Guzei and S. H. Choi, Org. Biomol. Chem. 2014, 12, 2641-2644. 